\title{
Second-Order Nonlinearity in Triangular Lattice Perforated Gold Film due to Surface Plasmas Resonance
}

\author{
Renlong Zhou, ${ }^{1}$ Xiaoshuang Chen, ${ }^{2}$ Yingyi Xiao, ${ }^{1}$ Bingju Zhou, ${ }^{1}$ Lingxi Wu, ${ }^{1}$ Xiaojuan Liu, ${ }^{1}$ \\ Yongyi Gao, ${ }^{1}$ and Jie Zhan ${ }^{1}$ \\ ${ }^{1}$ School of Physics and Electronic Science, Hunan University of Science and Technology, Xiangtan 411201, China \\ ${ }^{2}$ National Laboratory for Infrared Physics, Shanghai Institute of Technical Physics, Chinese Academy of Sciences, \\ Shanghai 200083, China \\ Correspondence should be addressed to Renlong Zhou; rlzhou@mail.sitp.ac.cn and Xiaoshuang Chen; xschen@mail.sitp.ac.cn
} Received 25 October 2013; Accepted 4 December 2013; Published 16 February 2014

Academic Editors: W. Hu, S. Jit, and F. Yue

Copyright (C) 2014 Renlong Zhou et al. This is an open access article distributed under the Creative Commons Attribution License, which permits unrestricted use, distribution, and reproduction in any medium, provided the original work is properly cited.

\begin{abstract}
We have studied the excitation second-order nonlinearity through a triangular lattice perforated gold film instead of square lattice in many papers. Under the excitation of surface plasmas resonance effect, the second order nonlinearity exists in the noncentrosymmetric split-ring resonators arrays. Reflection of fundamental frequency wave through a triangular lattice perforated gold film is obtained. We also described the second harmonic conversion efficiencies in the second order nonlinear optical process with the spectra. Moreover, the electric field distributions of fundamental frequency above the gold film region are calculated. The light propagation through the holes results in the enhancement of the second order nonlinearity including second harmonic generation as well as the sum (difference) frequency generation.
\end{abstract}

\section{Introduction}

Nonlinear optical responses have been subject of intensive studies due to the development of nanofabrication techniques [1-6]. Second harmonic generation (SHG) is a powerful tool for probing physical and structure properties of the interface and surface of materials [7-12]. The SHG in square lattice with different structures, such as double-hole arrays or semiconductor nanoparticles arrays, has also been well studied $[12,13]$. The underlying physical mechanism of SHG in metal nanoparticles has been explored numerically and experimentally [14-18]. The plasmonic oscillations of the conduction electrons inside the metal can induce the localized surface plasmon (SP) resonances. These localized surface plasmon (SP) resonances play important role in the process of nonlinear optical responses. The SHG from different SP resonance configurations such as split-ring resonators $[17,18]$, sharp metal tips $[19,20]$, metallodielectric multilayer structures [21], imperfect spheres [22, 23], and L-shaped and T-shaped nanoparticles $[9,24-26]$ has been investigated. The theory of second harmonic generation (SHG) in three-dimensional structures consisting of arbitrary distributions of metallic spheres made of centrosymmetric materials is developed by means of multiple scattering of electromagnetic multipole fields [27]. Other theoretical methods with various kinds of approaches for the SHG have been developed by using the finite difference time domain (FDTD) method [13].

In this work, we present the surface plasmas excitation of the SHG in three-dimensional triangular lattice structures consisting of noncentrosymmetric split-ring resonators. We obtained the reflection spectra of fundamental frequency wave through a triangular lattice structures consisting of split-ring resonators. We also described the $\mathrm{SH}$ conversion efficiencies in the second-order nonlinear optical process with the spectra of second harmonic generation field. We find that the SH conversion efficiencies of SHG signal are in the range of about $10^{-12}-10^{-14}$. The electric field distributions of fundamental frequency and second harmonic are also 
calculated by FDTD simulation. We have investigated the second-harmonic generation (SHG), sum frequency generation (SFG), and difference frequency generation (DFG). The enhanced SP resonance causes the increase of local secondorder nonlinearity.

\section{Theory and Method}

Second-order nonlinearity of split-ring resonators nanostructures has been studied with the classical theoretical method in [13]. We consider the three-dimensional triangular lattice structures consisting of gold split-ring resonators.

The permittivity $\varepsilon_{r}(\omega)$ of the gold split-ring resonators has the form

$$
\varepsilon_{r}(\omega)=1-\frac{\omega_{p}^{2}}{\omega(\omega+i \gamma)} .
$$

The $\gamma$ is collision frequency, and $w_{p}=\sqrt{e^{2} n_{0} / m_{e} \varepsilon_{0}}$ is the plasma frequency of gold. The plasma frequency and collision frequency are taken as $w_{p}=1.367 \times 10^{16} \mathrm{~s}^{-1}$ and $=6.478 \times$ $10^{13} \mathrm{~s}^{-1}$, respectively.

There are two computational loops for the calculations of the fundamental and second harmonic fields in the FDTD program. The FDTD method to calculate the first-order field at the fundamental frequency has been discussed in [13]. The FDTD approach is also applied for the numerical calculation of the second-order equations. If we consider the secondorder nonlinearity of the gold, the electric and magnetic field can be obtained as follows:

$$
\begin{aligned}
\frac{\partial B^{(2)}}{\partial t}= & -\nabla \times E^{(2)}, \quad \frac{\partial E^{(2)}}{\partial t}=c^{2} \nabla \times B^{(2)}-\frac{1}{\varepsilon_{0}} j^{(2)}, \\
j^{(2)}= & -i \omega p^{(2)}=-i \omega \varepsilon_{0}\left(\varepsilon_{r}-1\right) E^{(2)}+S^{(2)}, \\
& \frac{\partial j^{(2)}}{\partial t}=-\gamma j^{(2)}+\frac{e^{2} n_{0}}{m_{e}} E^{(2)}+S^{(2)}, \\
S^{(2)}= & \sum_{k} \frac{\partial}{\partial r_{k}}\left(\frac{j^{(1)} j^{(1)} k}{e n_{0}}\right) \\
& -\frac{e}{m_{e}}\left[\varepsilon_{0}\left(\nabla \cdot E^{(1)}\right) E^{(1)}+j^{(1)} \times B^{(1)}\right] .
\end{aligned}
$$

Here, $k$ represents the $x, y$, and $z$ coordinates. $J^{(1)}$ and $J^{(2)}$ represent the current density vectors of fundamental and harmonic waves, respectively. $E^{(1)}$ and $E^{(2)}, B^{(1)}$, and $B^{(2)}$ are the electric field and magnetic flux intensity vectors of fundamental and harmonic waves, respectively. $n_{0}$ is the ion density, $m_{e}$ is the electron mass, and $S^{(2)}$ is the nonlinear source of the plasma for second-order nonlinearity, respectively.

The structure of a triangular lattice perforated gold film is shown in Figure 1. The perfectly matched absorbing boundary conditions are employed at the bottom and top of the computational space along the $z$ direction, and the

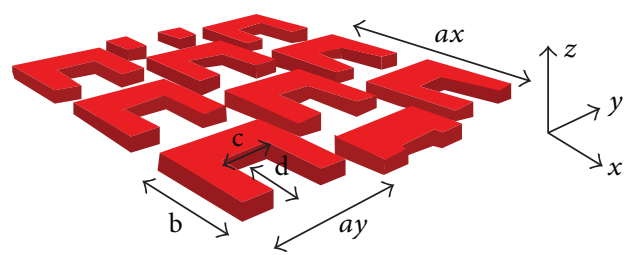

FIgURE 1: The structure of a triangular lattice structures consisting of gold split-ring resonators with the thickness $h=30.5 \mathrm{~nm}$ and the lattice periodic $a=a x=a y=305 \mathrm{~nm}$. There is the unit cell shape with $b=219 \mathrm{~nm}, c=131 \mathrm{~nm}$ and $d=97 \mathrm{~nm}$. The input light wave is polarized along the $y$ direction and propagates along the $z$ direction.

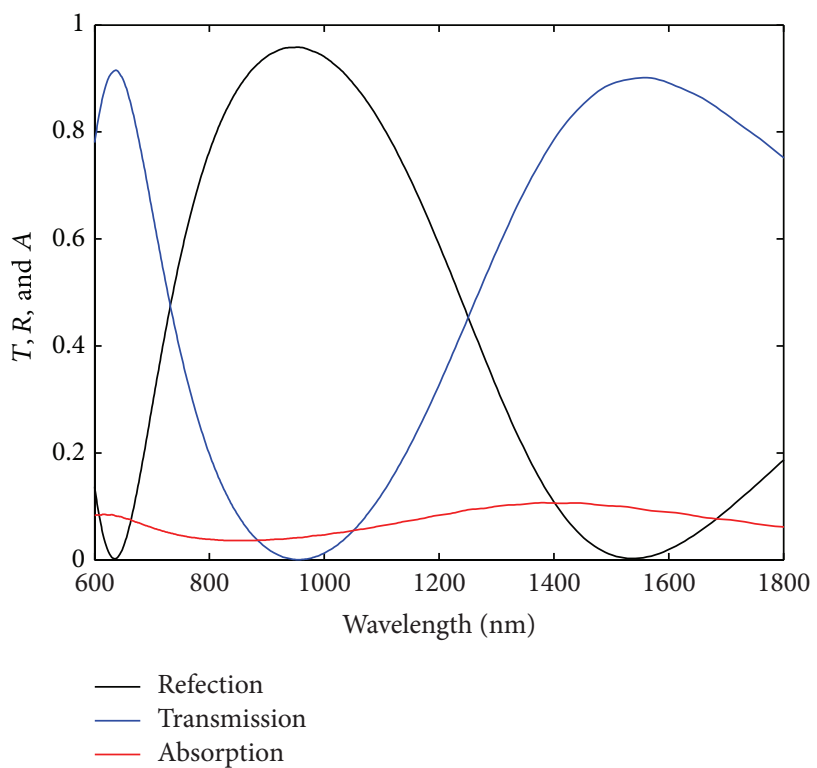

FIgURE 2: Reflection (transmission and absorption) spectra of fundamental frequency wave through a triangular lattice perforated gold film.

periodic boundary conditions are used on the boundaries of $x$ and $y$ directions. The incident wave is polarized along the $y$ direction and propagates along the $z$-axis. The triangular lattice structure consists of split-ring resonators with the thickness $h=30.5 \mathrm{~nm}$ and the lattice periodic $a=a x=$ ay $=305 \mathrm{~nm}$. The unit cell shape of split-ring resonator has $b=219 \mathrm{~nm}, c=131 \mathrm{~nm}$, and $d=97 \mathrm{~nm}$. The input light wave is polarized along the $y$ direction and propagates along the $z$ direction.

\section{Results and Discussions}

First, the normalized reflection (transmission and absorption) spectra of fundamental frequency wave through a triangular lattice perforated gold film are investigated here, and the calculation results are shown in Figure 2. There are two different SP resonance modes at the wavelengths $636 \mathrm{~nm}$ and $1548 \mathrm{~nm}$ for reflection spectra. These localized SP resonances at the wavelengths $636 \mathrm{~nm}$ and $1548 \mathrm{~nm}$ play important role in the process of nonlinear optical responses. 


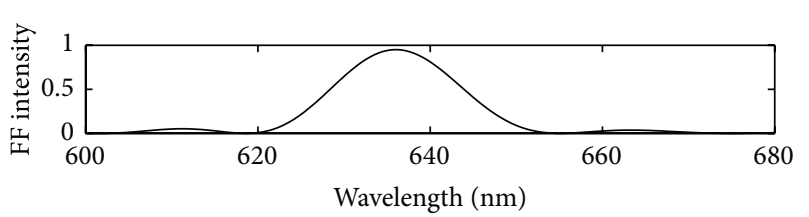

(a)

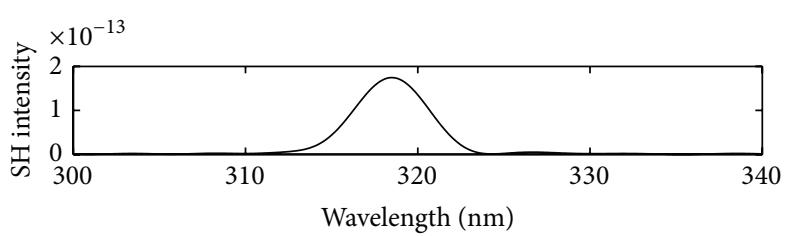

(b)

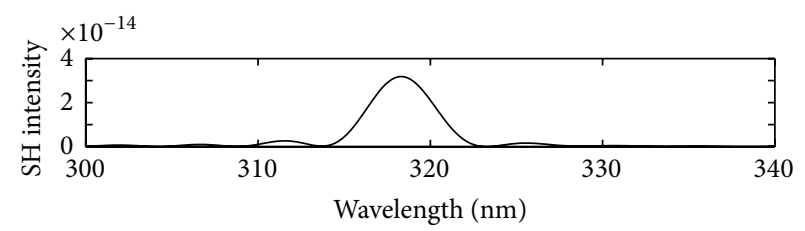

(c)

FIGURE 3: (a) The FTS for $E_{y}^{(1)}$ component of fundamental frequency field at the wavelength $636 \mathrm{~nm}$. The FTS for (b) $E_{x}^{(2)}$ and (c) $E_{y}^{(2)}$ component of second-harmonic generation at the wavelength $318 \mathrm{~nm}$.

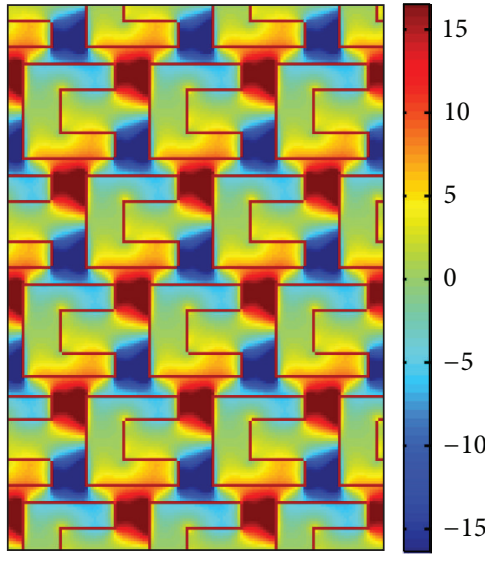

(a)

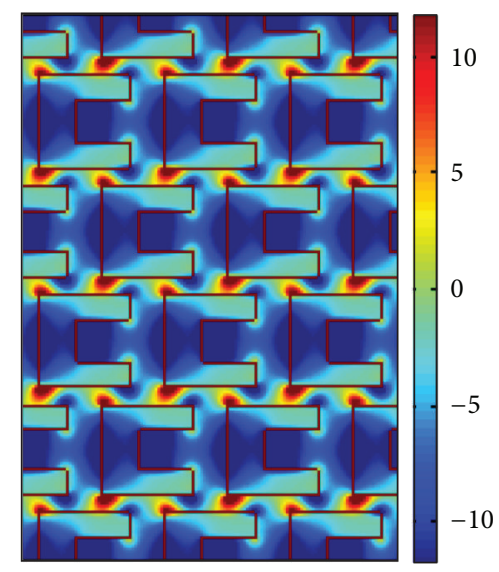

(b)

FIgURE 4: The electric field (a) $E_{x}^{(1)}$ and (b) $E_{y}^{(1)}$ distribution of fundamental frequency above the gold film region at wavelength $636 \mathrm{~nm}$, respectively.

The nonlinear optical responses from different SP resonance in split-ring resonators can result as different second-order nonlinearity.

To obtain the SHG with a triangular lattice perforated gold film patterns, the input light wave $E_{y}^{(1)}$ is polarized along the $y$ direction with wavelengths $\lambda_{1}$ or $\lambda_{2}$ :

$$
E_{y}^{(1)}=E_{0} \sin \left(\frac{2 \pi c t}{\lambda_{1,2}}\right),
$$

where $E_{0}$ is amplitude. We consider the wavelengths $\lambda_{1}=$ $636 \mathrm{~nm}$ or $\lambda_{2}=1548 \mathrm{~nm}$ in (3) in order to satisfy the transmission of the fundamental frequency waves, respectively.

When the continuous wave $E_{y}^{(1)}$ at wavelength $\lambda_{1}=$ $636 \mathrm{~nm}$ is incident through the triangular lattice perforated gold film, one can see Fourier transform spectrum (FTS) of fundamental frequency wave at the wavelength $636 \mathrm{~nm}$ in Figure 3(a). The FTS of the $E_{x}^{(2)}$ and the $E_{y}^{(2)}$ component of SHG at the wavelength $318 \mathrm{~nm}$ in Figures 3(b)-3(c), respectively, are also shown. To describe the $\mathrm{SH}$ conversion efficiencies in the second-order nonlinear optical process, the normalized SH intensity is defined as follows:

$$
\eta=\left|\frac{E^{(2)}\left(2 \omega_{0}\right)}{E^{(1)}\left(\omega_{0}\right)}\right|^{2},
$$

where $\omega_{0}$ is the freqency of the incident FF wave. The $x$ polarized SH conversion efficiencies are about $10^{-13}$ while the $y$-polarized SH conversion efficiencies are about $10^{-14}$ for the fundamental frequency wave at the wavelength $636 \mathrm{~nm}$ as shown in Figures 3(b)-3(c). The electric field distributions of $E_{x}^{(1)}$ and $E_{y}^{(1)}$ for fundamental frequency field above the gold film region at wavelengths $636 \mathrm{~nm}$ are also shown in Figures 4(a)-4(b), respectively. 


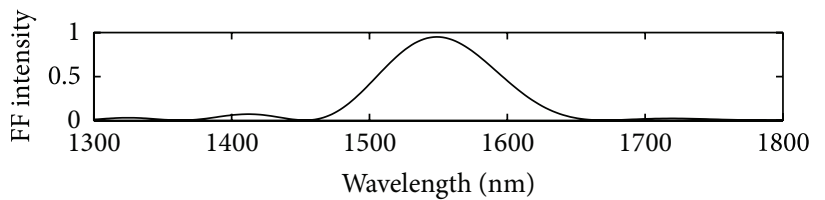

(a)

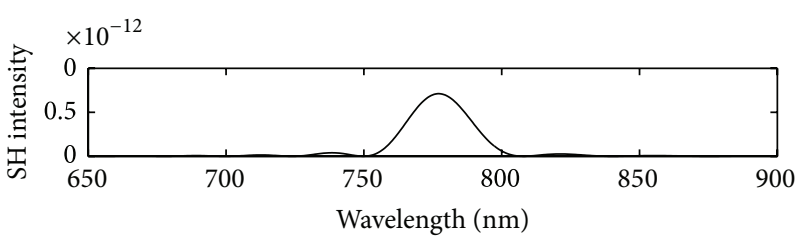

(b)

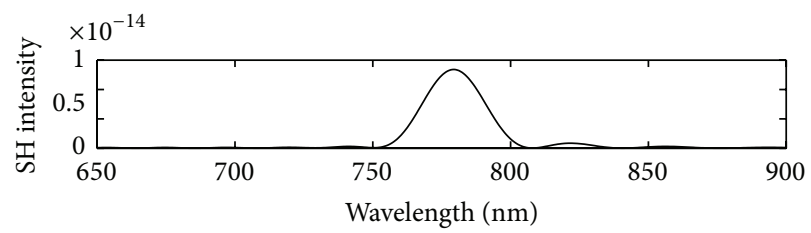

(c)

Figure 5: (a) The FTS for $E_{y}^{(1)}$ component of fundamental frequency wave at the wavelength $1548 \mathrm{~nm}$. The FTS for (b) $E_{x}^{(2)}$ and (c) $E_{y}^{(2)}$ component of second-harmonic generation at the wavelength $774 \mathrm{~nm}$.

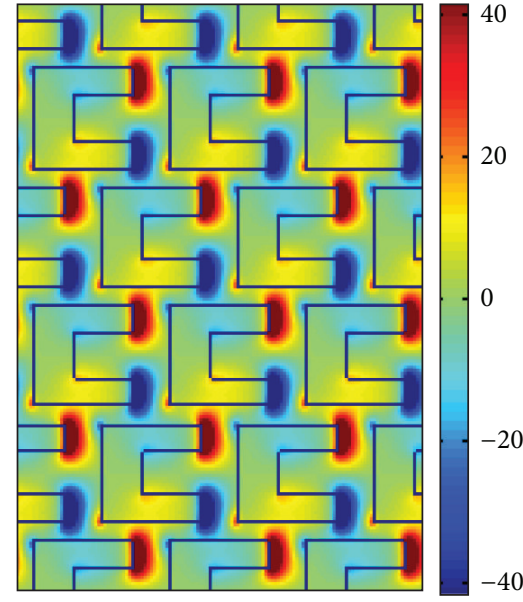

(a)

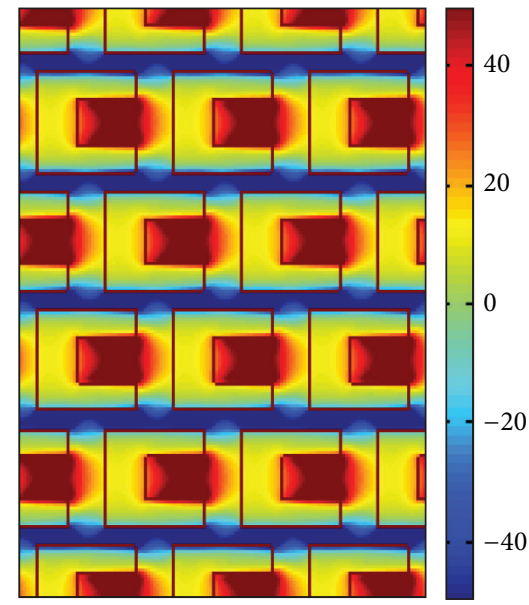

(b)

Figure 6: The electric field (a) $E_{x}^{(1)}$ and (b) $E_{y}^{(1)}$ distribution of fundamental frequency above the gold film region at wavelength $1548 \mathrm{~nm}$, respectively.

When the continuous wave $E_{y}^{(1)}$ at wavelengths $\lambda_{1}=$ $1548 \mathrm{~nm}$ is incident through the triangular lattice perforated gold film, one can see FTS of fundamental frequency wave at the wavelength $1548 \mathrm{~nm}$ in Figure 5(a). The FTS of the $E_{x}^{(2)}$ and the $E_{y}^{(2)}$ component of SHG at the wavelength $774 \mathrm{~nm}$ in Figures 5(b)-5(c), respectively, are also shown. The $x$-polarized SH conversion efficiencies are about $10^{-12}$ while the $y$-polarized SH conversion efficiencies are about $10^{-14}$ for the fundamental frequency wave at the wavelength $1548 \mathrm{~nm}$ as shown in Figures 5(b)-5(c). The electric field distribution of $E_{x}$ and $E_{y}$ for fundamental frequency field above the gold film region at wavelengths $1548 \mathrm{~nm}$ is also shown in Figures 6(a)-6(b), respectively.

To obtain the second-order nonlinearity including second-harmonic generation as well as the sum (difference) frequency generation of the triangular lattice gold film with split-ring resonators patterns, the input light wave
$E_{y}^{(1)}$ is polarized along the $y$ direction with two different wavelengths $\lambda_{1}$ and $\lambda_{2}$

$$
E_{y}^{(1)}=E_{0} \sin \left(\frac{2 \pi c t}{\lambda_{1}}\right)+E_{0} \sin \left(\frac{2 \pi c t}{\lambda_{2}}\right)
$$

where $E_{0}$ is amplitude. We consider two wavelengths $\lambda_{1}=$ $636 \mathrm{~nm}$ and $\lambda_{2}=1548 \mathrm{~nm}$ in (5).

When the continuous wave $E_{y}^{(1)}$ with two different wavelengths $636 \mathrm{~nm}$ and $1548 \mathrm{~nm}$ is incident through the triangular lattice perforated gold film, it is found that there are four peaks for second-order nonlinearity at the wavelengths $318 \mathrm{~nm}, 451 \mathrm{~nm}, 774 \mathrm{~nm}$, and $1080 \mathrm{~nm}$ in Figure 7, respectively. The second-order nonlinearity modes at wavelengths $318 \mathrm{~nm}$ and $774 \mathrm{~nm}$ are obtained from the SP modes of two 


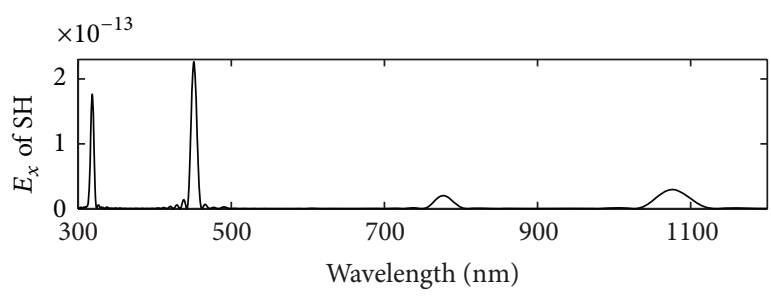

(a)

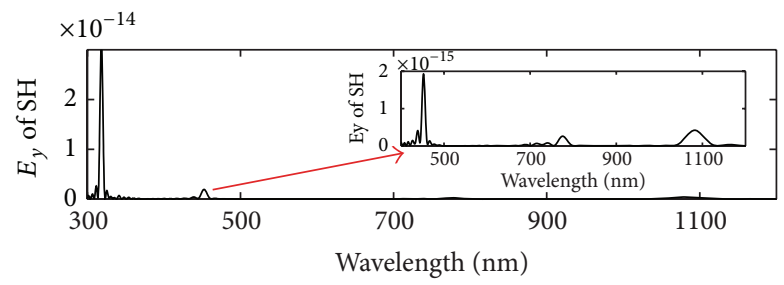

(b)

FIGURE 7: The FTS of second-order nonlinearity signals at the wavelength $318 \mathrm{~nm}, 451 \mathrm{~nm}, 774 \mathrm{~nm}$, and $1080 \mathrm{~nm}$ for the two continuous wave $E_{y}^{(1)}$ incidence at the wavelengths $\lambda_{1}=636 \mathrm{~nm}$ and $\lambda_{2}=1548 \mathrm{~nm}$, respectively.

incident continuous waves with wavelengths $636 \mathrm{~nm}$ and $1548 \mathrm{~nm}$ due to the SHG effect, respectively. The secondorder nonlinearity mode at wavelengths $451 \mathrm{~nm}$ is the sum frequency field signals for two incident continuous waves with wavelengths $636 \mathrm{~nm}$ and $1548 \mathrm{~nm}$. And the SH conversion efficiencies of sum-frequency field signal for two fundamental frequency wave incidence is about $10^{-13}$ or $10^{-14}$. The second-order nonlinearity mode at wavelength $1080 \mathrm{~nm}$ is the difference frequency field for two incident continuous waves with wavelengths $636 \mathrm{~nm}$ and $1548 \mathrm{~nm}$. The transmission of the fundamental light results from an enhancement of the local field. The strong local SP resonance induces an increase of the four second-order nonlinearity signals. The enhancement of the second-order nonlinearity signals include second-harmonic generation as well as the sum (difference) frequency generation.

\section{Conclusions}

Unlike SGH in square lattice perforated gold film in many papers, we have studied the excitation of second-order nonlinearity signals through a triangular lattice perforated gold film due to surface plasmas resonance effect. Based on the FDTD method, the SH conversion efficiencies in the secondorder nonlinear optical process are studied. The electric field distributions of fundamental frequency are calculated. The second-order nonlinearity phenomenon including secondharmonic generation as well as the sum (difference) frequency generation is shown in our paper.

\section{Conflict of Interests}

The authors declare that there is no conflict of interests regarding the publication of this paper.

\section{Acknowledgments}

This work was supported by the National Natural Science Foundation of China under Grants 11247003 and 51175172. The authors would especially like to thank Dr. Yong Zeng for the discussion of FDTD simulations.

\section{References}

[1] U. Kreibig and M. Vollmer, Optical Properties of Metal Clusters, Springer, Berlin, Germany, 1995.

[2] T. F. Heinz, Nonlinear Surface Electromagnetic Phenomena, edited by H. E. Ponath and G. I. Stegeman, chapter 5, NorthHolland, Amsterdam, The Netherlands, 1991.

[3] H. Wang, E. Yan, E. Borguet, and K. B. Eisenthal, "Second harmonic generation from the surface of centrosymmetric particles in bulk solution," Chemical Physics Letters, vol. 259, no. 1-2, pp. 15-20, 1996.

[4] J. I. Dadap, J. Shan, K. B. Eisenthal, and T. F. Heinz, "Secondharmonic Rayleigh scattering from a sphere of centrosymmetric material," Physical Review Letters, vol. 83, no. 20, pp. 4045-4048, 1999.

[5] G. Gonella and H.-L. Dai, "Determination of adsorption geometry on spherical particles from nonlinear Mie theory analysis of surface second harmonic generation," Physical Review B, vol. 84, no. 12, Article ID 121402, 5 pages, 2011.

[6] J. I. Dadap, J. Shan, and T. E. Heinz, "Theory of optical second-harmonic generation from a sphere of centrosymmetric material: small-particle limit," Journal of the Optical Society of America B, vol. 21, no. 7, pp. 1328-1347, 2004.

[7] L. Wang, W. Hu, J. Wang et al., "Plasmon resonant excitation in grating-gated AlN barrier transistors at terahertz frequency," Applied Physics Letters, vol. 100, no. 12, Article ID 123501, 2012.

[8] D. Hu, L. Wang, X. S. Chen et al., "Room-temperature plasmonic resonant absorption for grating-gate GaN HEMTs in far infrared terahertz domain," Optical and Quantum Electronics, vol. 45, no. 7, pp. 713-720, 2013.

[9] G. Li, X. Chen, B. Ni et al., "Fractal H-shaped plasmonic nanocavity," Nanotechnology, vol. 24, no. 20, Article ID 205702, 2013.

[10] G. Li, X. Chen, O. Li et al., "A novel plasmonic resonance sensor based on an infrared perfect absorber," Journal of Physics D, vol. 45, no. 20, Article ID 205102, 2012.

[11] J. U. Fürst, D. V. Strekalov, D. Elser et al., "Naturally phasematched second-harmonic generation in a whispering-gallerymode resonator," Physical Review Letters, vol. 104, no. 15, Article ID 153901, 2010.

[12] J. Butet, G. Bachelier, I. Russier-Antoine, C. Jonin, E. Benichou, and P.-F. Brevet, "Interference between selected dipoles and octupoles in the optical second-harmonic generation from spherical gold nanoparticles," Physical Review Letters, vol. 105, no. 7, Article ID 077401, 2010. 
[13] Y. Zeng, W. Hoyer, J. Liu, S. W. Koch, and J. V. Moloney, "Classical theory for second-harmonic generation from metallic nanoparticles," Physical Review B, vol. 79, no. 23, Article ID 235109, 2009.

[14] C. M. Soukoulis, S. Linden, and M. Wegener, "Physics. Negative refractive index at optical wavelengths," Science, vol. 315, no. 5808, pp. 47-49, 2007.

[15] V. M. Shalaev, "Optical negative-index metamaterials," Nature Photonics, vol. 1, no. 1, pp. 41-48, 2007.

[16] J. Valentine, S. Zhang, T. Zentgraf et al., "Three-dimensional optical metamaterial with a negative refractive index," Nature, vol. 455, no. 7211, pp. 376-379, 2008.

[17] M. W. Klein, C. Enkrich, M. Wegener, and S. Linden, "Secondharmonic generation from magnetic metamaterials," Science, vol. 313, no. 5786, pp. 502-504, 2006.

[18] M. W. Klein, M. Wegener, N. Feth, and S. Linden, "Experiments on second- and third-harmonic generation from magnetic metamaterials," Optics Express, vol. 15, no. 8, pp. 5238-5247, 2007.

[19] Y. Zeng and J. V. Moloney, "Volume electric dipole origin of second-harmonic generation from metallic membrane with none entro symmetric patterns," Optics Letters, vol. 34, no. 18, pp. 2844-2846, 2009.

[20] C. C. Neacsu, G. A. Reider, and M. B. Raschke, "Secondharmonic generation from nanoscopic metal tips: symmetry selection rules for single asymmetric nanostructures," Physical Review B, vol. 71, no. 20, Article ID 201402, 2005.

[21] M. C. Larciprete, A. Belardini, M. G. Cappeddu et al., "Second-harmonic generation from metallodielectric multilayer photonic-band-gap structures," Physical Review A, vol. 77, no. 1, Article ID 013809, 2008.

[22] J. Shan, J. I. Dadap, I. Stiopkin, G. A. Reider, and T. F. Heinz, "Experimental study of optical second-harmonic scattering from spherical nanoparticles," Physical Review A, vol. 73, no. 2, Article ID 023819, 2006.

[23] J. Nappa, G. Revillod, I. Russier-Antoine, E. Benichou, C. Jonin, and P. F. Brevet, "Electric dipole origin of the second harmonic generation of small metallic particles," Physical Review B, vol. 71, no. 16, Article ID 165407, 2005.

[24] S. Kujala, B. K. Canfield, M. Kauranen, Y. Svirko, and J. Turunen, "Multipolar analysis of second-harmonic radiation from gold nanoparticles," Optics Express, vol. 16, no. 22, pp. 17196-17208, 2008.

[25] H. Husu, B. K. Canfield, J. Laukkanen et al., "Chiral coupling in gold nanodimers," Applied Physics Letters, vol. 93, no. 18, Article ID 183115, 2008.

[26] B. K. Canfield, H. Husu, J. Laukkanen et al., "Local field asymmetry drives second-harmonic generation in noncentrosymmetric nanodimers," Nano Letters, vol. 7, no. 5, pp. 12511255, 2007.

[27] J. Xu and X. Zhang, "Second harmonic generation in threedimensional structures based on homogeneous centrosymmetric metallic spheres," Optics Express, vol. 20, no. 2, pp. 16681684, 2012. 

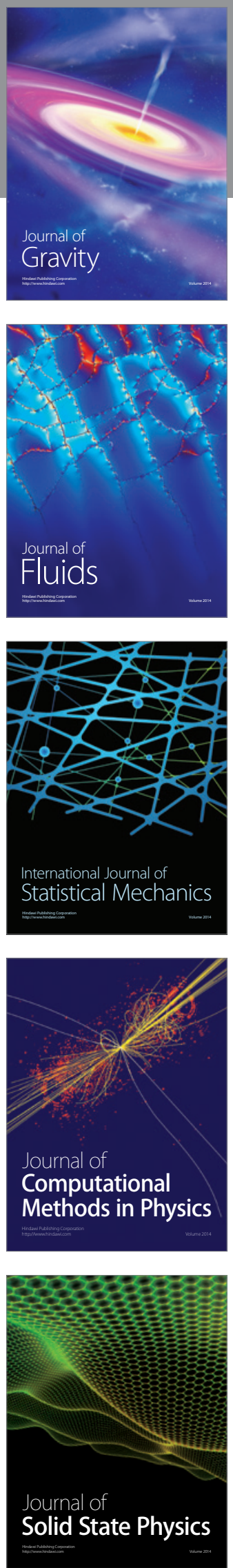

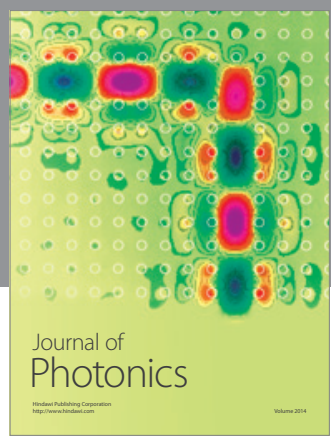

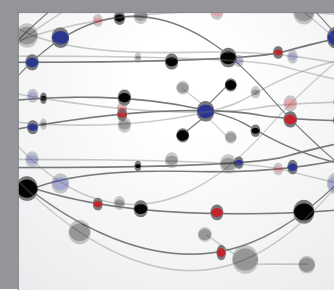

The Scientific World Journal

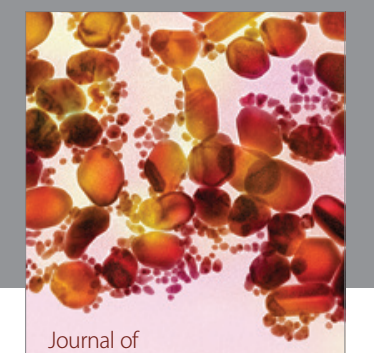

Soft Matter
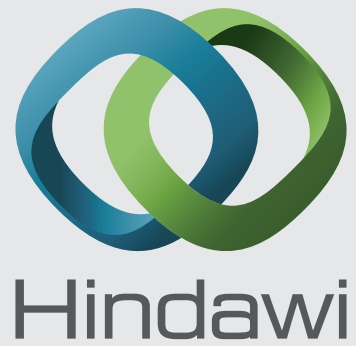

Submit your manuscripts at

http://www.hindawi.com
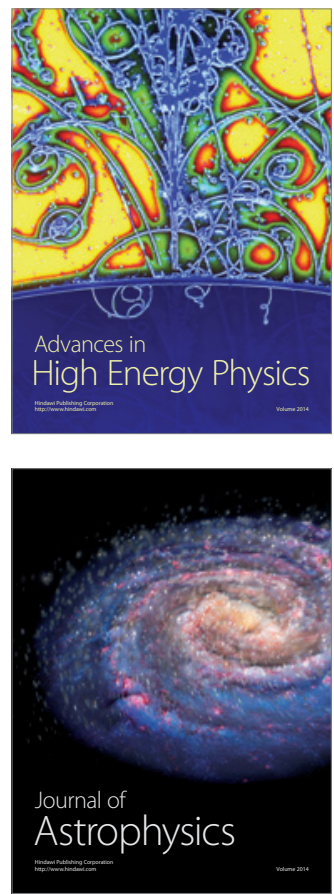
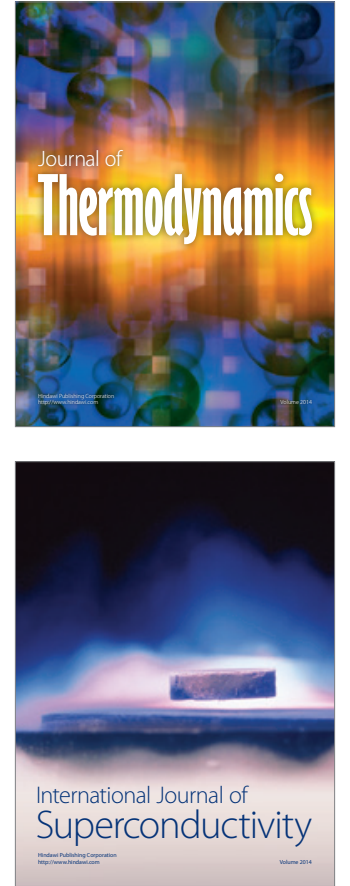
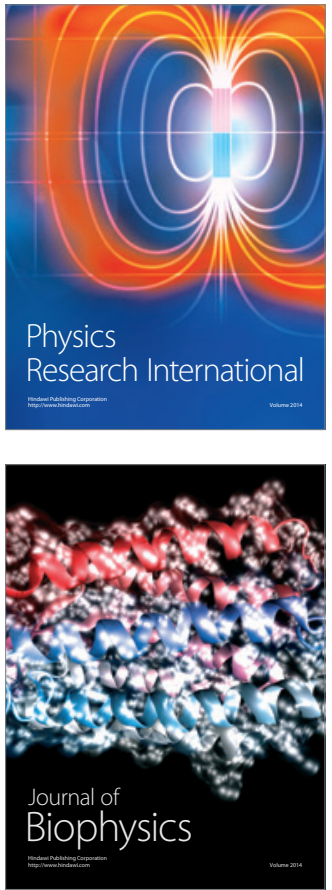
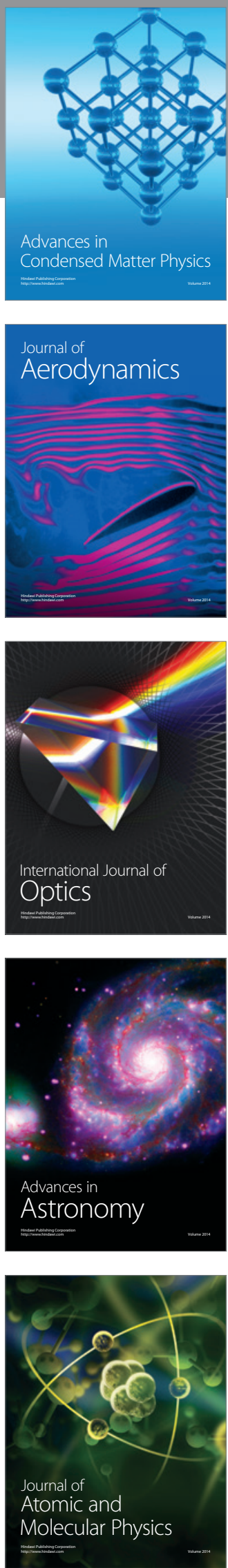